\title{
Thermoelectric properties of elemental metals from first-principles electron-phonon coupling
}

\author{
Bin Xu $\odot,{ }^{1, *}$ Marco Di Gennaro $\odot,{ }^{2}$ and Matthieu J. Verstraete ${ }^{2}$ \\ ${ }^{1}$ Jiangsu Key Laboratory of Thin Films, School of Physical Science and Technology, Soochow University, Suzhou 215006, China \\ ${ }^{2}$ Nanomat, Q-Mat, CESAM, and European Theoretical Spectroscopy Facility, Université de Liège, B-4000 Liège, Belgium
}

(Received 16 July 2020; revised 31 August 2020; accepted 28 September 2020; published 20 October 2020)

\begin{abstract}
The Seebeck coefficient is one of the key ingredients in thermoelectric properties, and it is often calculated based simply on the electronic band structure, within the frame of Boltzmann's transport theory and the constant relaxation time approximation. Despite the simplicity and popularity of this approximation, its validity is not fully justified even in lightly doped semiconductors, and it breaks down completely in metals. On the other hand, more sophisticated first-principles approaches are available but require the computation of the full electronphonon coupling. Here, we demonstrate with several simple (alkali and noble) metals viz., $\mathrm{Li}, \mathrm{Na}, \mathrm{K}, \mathrm{Cu}, \mathrm{Ag}$, $\mathrm{Au}$, and $\mathrm{Pt}$, that the variational approach based on ab initio couplings can reproduce experimental Seebeck coefficients quantitatively, whereas the constant relaxation time approximation yields significant quantitative discrepancies and often fails to predict the correct sign. Calculations of the electrical resistivity of these metals via the variational approach are also reported.
\end{abstract}

DOI: 10.1103/PhysRevB.102.155128

\section{INTRODUCTION}

Thermoelectric (TE) effects hold great promise in energy saving and efficiency applications, through the ability to convert (waste) heat into electricity or vice versa. The Seebeck effect relates a thermal gradient to a voltage difference and is directly related to the entropy flow per particle, making it a sensitive probe of phase transitions and thermodynamics (reviewed, e.g., in Ref. [1]). Many efforts [2,3] have been devoted to improve the TE figure of merit (ZT), requiring a material to have high power factor (product of electrical conductivity $\sigma$ and square of Seebeck coefficient $S^{2}$ ) and low thermal conductivity. However, intriguing interdependencies among these transport properties make systematic improvement very challenging [4]. To find new TE materials with better performance, in addition to experiments, computations have played an important role in interpreting the TE behavior, understanding the underlying mechanism, and even predicting TE properties for new materials via density functional theory (DFT), high throughput [5-7], or machine learning techniques [8-10].

Accurate calculation of TE properties is vital in the intelligent materials search framework, either for a detailed understanding of the physics or to guarantee high-fidelity data. The most widely used first-principles computational method is to solve the Boltzmann transport equation (BTE) by the constant relaxation time approximation (CRTA) [11-13]. This approximation is not applicable rigorously even for the simplest case, i.e., free electrons with a spherical Fermi surface since the electron-phonon scattering is anisotropic. However the CRTA has the strong advantage of efficiency-it only needs band energies and derivatives as input-and it works surprisingly well, even beyond its formal range of applicabil-

*binxu19@suda.edu.cn ity in many cases, for instance in Refs. [14,15]. For lightly doped semiconductors, the current state of the art is the full wave vector dependent solution of Boltzmann's equation [16-18], using an electron-phonon coupling (EPC) scattering kernel calculated from first principles and making an effective relaxation time approximation: Each electron state has a single averaged scattering time, in the mean field of all other electrons in equilibrium. This approach has only started to be applied to transport in metals recently [19-21], in part because most metal Fermi surfaces are much larger than the pockets found in lightly doped semiconductors. This implies heavier numerical grid integrations involving all points in the Brillouin zone (BZ), instead of a small fraction. A simple variational approach (VA) was developed by Allen in the 1970s [22] based on spatial harmonics of the Fermi surface. Allen's method has been extended by Eiguren and Gurtubay [23] to solve an explicit Helmholtz equation on the numerical Fermi surface (FS), but we will use the standard approach, in its fully inelastic expression.

In 2014 we showed that the CRTA yields the wrong sign for the Seebeck coefficient in lithium, whereas the VA works very well [24]. Lithium should have electrons as the majority charge carriers, and this is borne out by Hall effect measurements $[25,26]$. One would usually expect $S<0$, which the CRTA produces, but in $\mathrm{Li}$ the true $S$ is positive and increases with temperature. It remains an open question whether the failure of CRTA in lithium is only a special case or if it occurs widely in other materials: In the transition metal series there are many instances of both positive and negative $S$.

The Seebeck coefficients of $\mathrm{Cu}, \mathrm{Ag}, \mathrm{Au}$, and $\mathrm{Pt}$ have recently been studied by Kou and Akai using the KorringaKohn-Rostoker coherent potential approximation (KKRCPA) method, which yields the correct sign with some quantitative discrepancies [27]. The same method was also adopted to predict the behavior of $S$ under high pressure and temperature for $\mathrm{Pt}$ [28]. 
In this paper, we extend the comparison between CRTA and VA to other elemental metals, including sodium and potassium (alkali metals with negative $S$ ), and four noble metals, viz., copper, silver, gold, and platinum, which have filled $d$ bands, $s$-like nearly-free-electron (NFE) conduction, but for which $S>0$ in $\mathrm{Cu}, \mathrm{Ag}$, and $\mathrm{Au}$, and $S<0$ in Pt. The choice of elements covers a variety of cases to extract insight on the validity of the two computational approaches. We find that the CRTA fails often in metals, where the details of the band dispersion as well as the varying strength of the EPC both matter in detail for the value and even the sign of $S$. The $\mathrm{VA}$ is robust and efficient, requiring a one-shot FS integral for a closed form solution of the BTE.

\section{COMPUTATIONAL DETAILS}

The electronic band structures, electronic velocities, density of states (DOS), phonon dispersion, and EPC matrix elements are calculated within density functional theory (DFT) and density functional perturbation theory (DFPT) [29,30], carried out using the ABINIT package [31-33]. The exchange and correlation functional is treated with the generalized gradient approximation and the Perdew-BurkeErnzerhof functional (GGA-PBE). See the Appendix for more details. For each studied metal, an unshifted $24 \times 24 \times 24$ kpoint grid and $12 \times 12 \times 12$ q-point grid are employed, ensuring good convergence for transport properties. A $36 \times$ $36 \times 36$ k-point grid and $12 \times 12 \times 12$ q-point grid yield nearly the same resistivity and Seebeck coefficient for Pt, which has the most complex Fermi surface among the studied cases. The EPC matrix elements on the coarse $\mathbf{q}$ grid are Fourier interpolated onto the dense $\mathbf{k}$ grid. We note that the small FS pockets in doped semiconductors makes $k$-point convergence more stringent than in metals, and the VA being a full integral over the FS is less sensitive than individual state lifetimes. The plane-wave basis functions with kinetic energies up to 20 Hartree are used in alkali metals and 25 Hartree in noble metals. Test calculation with $40 \mathrm{Ha}$ of cutoff energy for Au yields a very similar result. We also examine the effect of spin-orbit coupling, for heavy elements such as Au and Pt. The band shape can be slightly altered, as compared in Ref. [34] for Au, and in certain compounds, such as bismuth [35] or lead [36], the phonons can be altered as well.

The Seebeck coefficients are calculated using at least two methods, both within the frame of Boltzmann's transport theory. The first method takes the CRTA solution to the BTE, as implemented in the BOLTZTRAP code [11,12]. Within the
CRTA, $S$ is independent of the lifetime chosen. The second method is the VA, adopting the lowest-order variational approximation (LOVA) to the Boltzmann transport equation, as derived by Allen [37]. In our work only electron phonon scattering is considered, which is the main mechanism and has a strong temperature dependence in pure metals, and the Fermi smearing effect is fully considered. Via VA, the electrical resistivity is also calculated. Details of both methods are described in Appendix A. A significant advantage of the VA is that the full distribution of occupations is in a realistic, out-of-equilibrium state. In standard relaxation time approximations, each lifetime is calculated presuming equilibrium occupations for all other states, as a single electron is excited and then relaxes back (exponentially) to its ground state. In the VA an effective steady state is posited with a functional form for the occupation departure from equilibrium, giving full transport coefficients in a single integral. One limitation is for very small Fermi surfaces, where dense wave-vector grids must be used. This problem is present in all EPC methods, but RTA lifetimes are always well defined, even for $T=0$ in a semiconductor with the chemical potential in the gap. As shown in the Appendix, the VA functional forms by Allen include a $1 / N\left(\epsilon_{F}\right)$ factor, which will fluctuate strongly near a band edge and is not defined in the gap.

\section{RESULTS AND DISCUSSION}

For each metal, the ambient-condition crystal structure is considered in this work, i.e., body centered cubic (bcc) for the alkali metals and face centered cubic (fcc) for the noble metals. These structures also turn out to be the ones that occur in the widest temperature range. Lithium and sodium are known to transform to a closed-packed $9 R$ phase at low temperatures $(<\sim 77 \mathrm{~K}$ for $\mathrm{Li}$ and $<\sim 35 \mathrm{~K}$ for $\mathrm{Na}$ ) [42].

DFT optimized lattice constants are used for calculations of band structures and transport properties [43]. As listed in Table I, the calculated values agree well with experiments measured at room temperature, with errors all being less than $2 \%$. GGA typically overestimates lattice constants, which is the case for $\mathrm{Cu}, \mathrm{Au}$, and $\mathrm{Pt}$, but interestingly, underestimation is found for $\mathrm{Li}, \mathrm{Na}, \mathrm{K}$, and $\mathrm{Ag}$. Note that thermal expansion is not taken into account in the present study. Test calculations on Au with slightly larger lattice constant (corresponding to $1000 \mathrm{~K}$ ) show that the influence on transport properties is quite small, e.g., $S$ at $1200 \mathrm{~K}$ is only $9 \%$ smaller than that of the relaxed lattice constant.

TABLE I. Calculated lattice constant (in $\AA$ ), transport electron phonon coupling constant $\lambda_{\text {tr }}$, and electron-phonon mass enhancement parameter $\lambda$ for the alkali and noble metals. Experimental and other reported values are also listed.

\begin{tabular}{lccccccc}
\hline \hline & $\mathrm{Li}$ & $\mathrm{Na}$ & $\mathrm{K}$ & $\mathrm{Cu}$ & $\mathrm{Ag}$ & $\mathrm{Au}$ & $\mathrm{Pt}$ \\
\hline$a$ (This work) & 3.436 & 4.199 & 5.123 & 3.655 & 4.061 & 4.104 & 3.945 \\
$a$ (Expt. at RT) [26,38] & 3.491 & 4.225 & 5.225 & 3.597 & 4.079 & 4.065 & 3.912 \\
& & & & & & & \\
& & & & & & \\
$\lambda_{\text {tr }}$ (This work) & 0.236 & 0.142 & 0.071 & 0.126 & 0.128 & $0.137 / 0.219$ (SOC) & $0.534 / 0.700(\mathrm{SOC})$ \\
$\lambda_{\text {tr }}$ (Ref. [39]) & 0.35 & 0.14 & 0.11 & 0.13 & 0.12 & 0.15 & 0.66 \\
$\lambda$ (This work) & 0.411 & 0.220 & 0.101 & 0.137 & 0.150 & $0.193 / 0.220(\mathrm{SOC})$ & $0.699 / 0.461(\mathrm{SOC})$ \\
$\lambda$ (Ref. [21,39-41]) & $0.4 \pm 0.1$ & $0.16 \pm 0.03$ & $0.13 \pm 0.03$ & $0.15 \pm 0.03$ & $0.13 \pm 0.04$ & $0.17 \pm 0.05$ & $0.31,0.559,0.612,0.58$ \\
\hline \hline
\end{tabular}



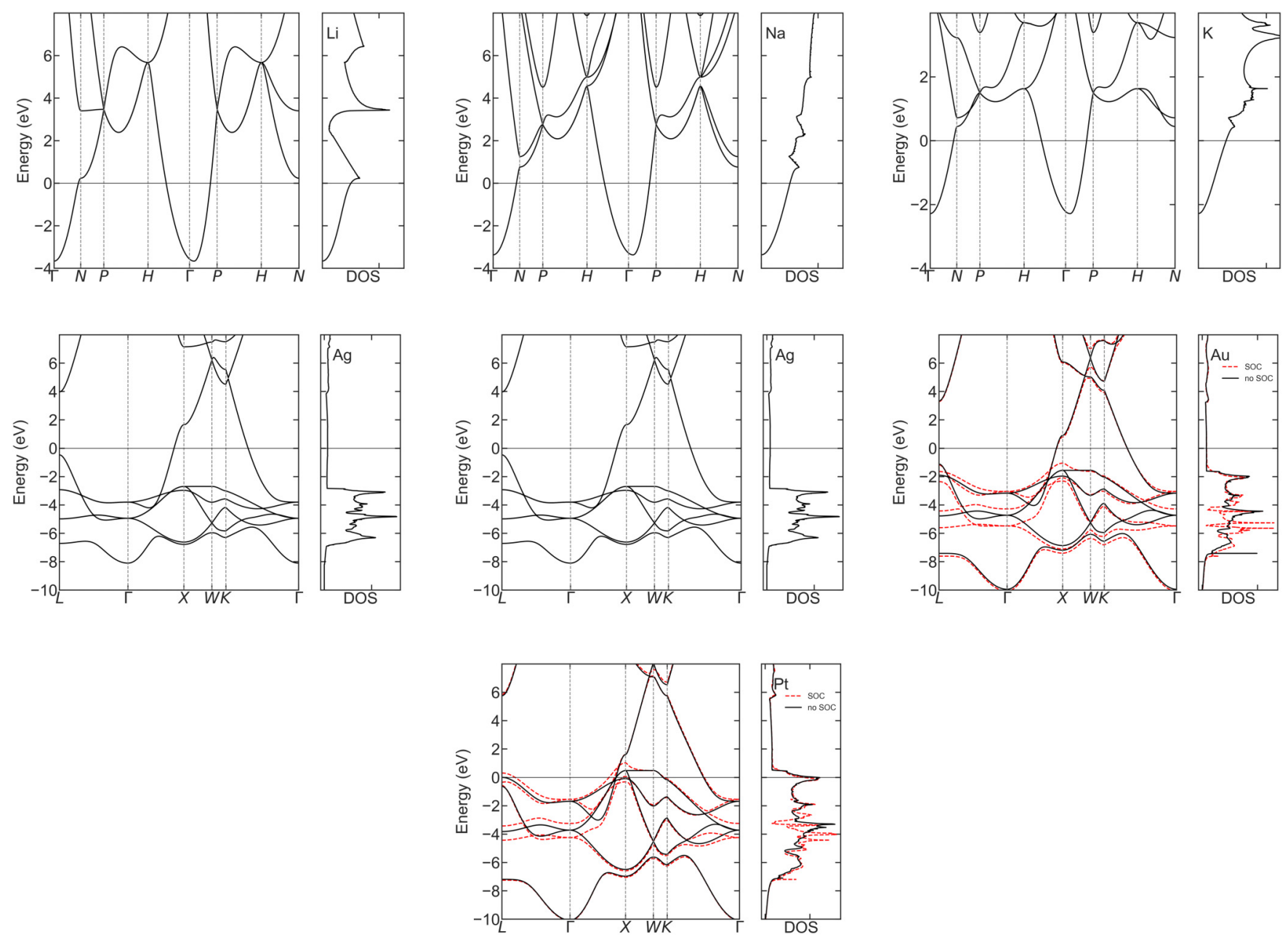

FIG. 1. Calculated band structure and density of states (DOS) for Li, Na, K, Cu, Ag, Au, and Pt. For heavy elements Au and Pt, results with spin orbit coupling (SOC) are also included.

We first examine the electronic band structures, which are well known in the literature but essential to understand the transport properties. In particular, band energies are the only ingredient for the CRTA approach. The alkali metals have one $s$ valence electron, thus NFE-like behavior is expected. This is a good approximation for $\mathrm{Na}$ and $\mathrm{K}$, since the Fermi energy $\left(\epsilon_{F}\right)$ crosses a band with a parabolic shape, as shown in Fig. 1. But in $\mathrm{Li}, \epsilon_{F}$ is very close to a distortion of the NFE band structure, which corresponds to the spherical Fermi surface bulging towards the $N$ point on the boundary of the first $\mathrm{BZ}$ (Fig. 3). In Fig. 2, it clearly shows that the DOS in Li has a significant increase near $\epsilon_{F}$, whereas it is close to a linear relationship for $\mathrm{Na}$ and $\mathrm{K}$ (in the tail of a square root DOS).

For $\mathrm{Cu}, \mathrm{Ag}$, and $\mathrm{Au}$, the outermost valence electron is also one $s$ electron in addition to the fully filled $d$ orbitals, and the bands near $\epsilon_{F}$ resemble the parabolic band in alkali metals, but with two kinks, one above and one below the $\epsilon_{F}$, which correspond to the Fermi surface touching the Brillouin zone boundary at $\mathrm{X}$ and $\mathrm{L}$ points (Fig. 3), respectively. The electron filling of the Fermi sphere is the combination of the influence of the inner $d$ states and the lattice constant, causing the DOS to deviate from the NFE picture (Fig. 2). The energy dependence of the DOS near $\epsilon_{F}$ is monotonic decreasing for $\mathrm{Cu}$ but more complex for $\mathrm{Ag}$ and $\mathrm{Au}$. On the other hand, $\mathrm{Pt}$ atoms have $5 d^{9}$ and $6 s^{1}$ electrons, yielding an $\epsilon_{F}$ crossing both the $d$ and $s$ bands. Its DOS at $\epsilon_{F}$ is close to a maximum and decreases faster on the higher energy side. The Fermi surface of $\mathrm{Pt}$ is more complex: The Fermi surface of the $s$ electrons is significantly distorted from the spherical shape (red color in Fig. 3), and the $d$-electron Fermi surface is holelike (a small hole pocket in yellow and a complex hole network in green). Purely based on the DOS, holelike transport behavior may be expected from $\mathrm{Cu}$ and $\mathrm{Pt}$, but both metals have negative Hall coefficients so that the majority charge carriers are still electrons. For Au and Pt, band structures including SOC are in good agreement with former DFT calculations [34,44]. SOC has a noticeable effect on the band structure, in particular for the filled bands.

The other ingredient of electron phonon coupling-phonon dispersion-is shown in Appendix B (Fig. 8). The DFPT calculated phonon dispersion curves are in reasonably good agreement with experiments, with a maximum error over all elements of less than $9 \%$ for the highest frequency. The slight overestimation in $\mathrm{Li}$ and $\mathrm{K}$ is likely due to the smaller lattice constants from DFT compared with measurements, and likewise, the underestimation in $\mathrm{Au}$ can be related to the larger lattice constant adopted in the phonon calculation or the neglect of SOC. Excepting Pt, a clear trend can be seen 

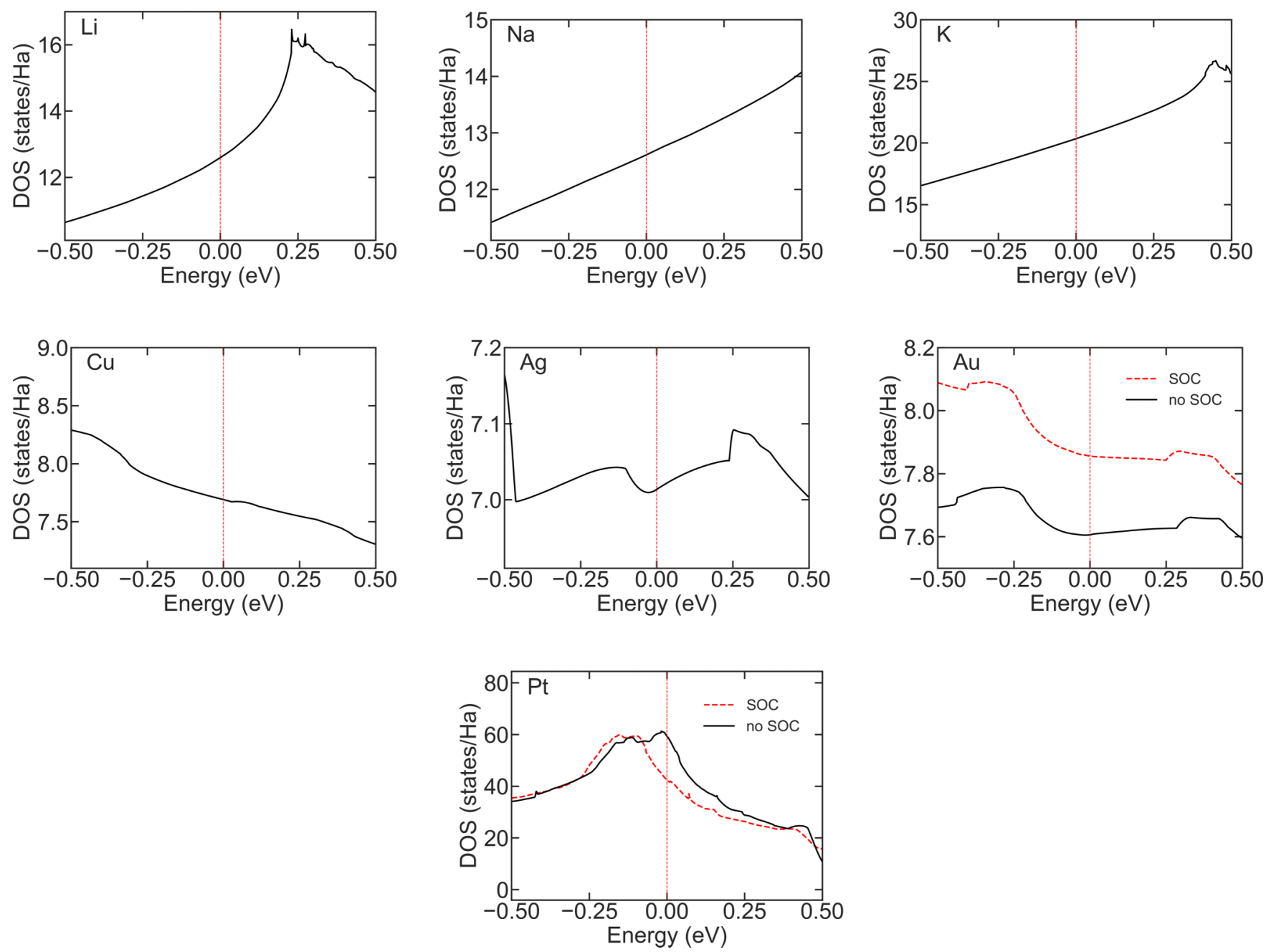

FIG. 2. Calculated density of states near the Fermi energy for $\mathrm{Li}, \mathrm{Na}, \mathrm{K}, \mathrm{Cu}, \mathrm{Ag}, \mathrm{Au}$, and Pt. For heavy elements $\mathrm{Au}$ and Pt, results with spin orbit coupling (SOC) are also included.

in the alkali and noble metals; for each group the phonons become softer with increasing atomic number, maintaining a similar shape of the dispersion curves. SOC is found to slightly upshift the phonon frequencies, yielding improved agreement for $\mathrm{Au}$.

The transport properties are determined by the electron phonon interactions, but it is nontrivial to understand EPC simply based on the Fermi surfaces and phonon dispersions; the transport electron phonon coupling constant [Eq. (A11) in Appendix A] characterizes the integrated average strength of EPC related to transport properties. In alkali metals, as shown in Table I, $\lambda_{\text {tr }}$ decreases monotonically from Li to $\mathrm{K}$ because of improved Fermi surface nesting in $\mathrm{Li}$ and the harder phonons. On the other hand, in noble metals, $\lambda_{\text {tr }}$ increases slightly from $\mathrm{Cu}$ to $\mathrm{Au}$, related to Fermi nesting phonon frequency changes. Note that $\lambda_{\text {tr }}=0.14$ for $\mathrm{Cu}$ was reported by Savrasov and Savrasov [45], which is quite close to our result. The other noble metal Pt has the largest transport EPC constant among the studied metals, contributed by its complex Fermi surface that allows more effective Fermi nesting. For heavier elements $\mathrm{Au}$ and $\mathrm{Pt}, \lambda_{\text {tr }}$ is apparently larger when including SOC. We also listed the estimated values based on the experimental resistivities [39], which are generally consistent with our predictions. The electron-phonon mass enhancement parameter $\lambda$ is also calculated and compared with literature values in Table I.

Now let us turn to the temperature dependence of the electrical resistivity $\rho$ (Figs. 4 and 9). For the alkali metals, only the bcc structure is considered in our calculations, which should be compared with experiments. Also note that $\rho$ is calculated with the variational approach [Eq. (A14) in Appendix A], which does not require any empirical parameter or fitting to experimental data. The CRTA approach is not adopted for $\rho$ because of the undetermined relaxation time: The intrinsic electron temperature variation of $\rho$ in the CRTA is almost negligible (less than 1 part per thousand over $1500 \mathrm{~K}$ ), and the full $T$ dependency is due to the variation of the effective electron lifetime following EPC. The VA calculated results are in good agreement with the measured data [46] for $\mathrm{Li}$; in particular the temperature dependence is well reproduced. The DFT value in the elastic resistivity formula initially used by Allen and then Savrasov is linear at high $T$ by construction, but the exponent in our inelastic formula can vary, which is visible in the plots for Pt and K. For Na the agreement is good up to room temperature, then the measured $\rho$ increases faster. For K, the agreement holds only 

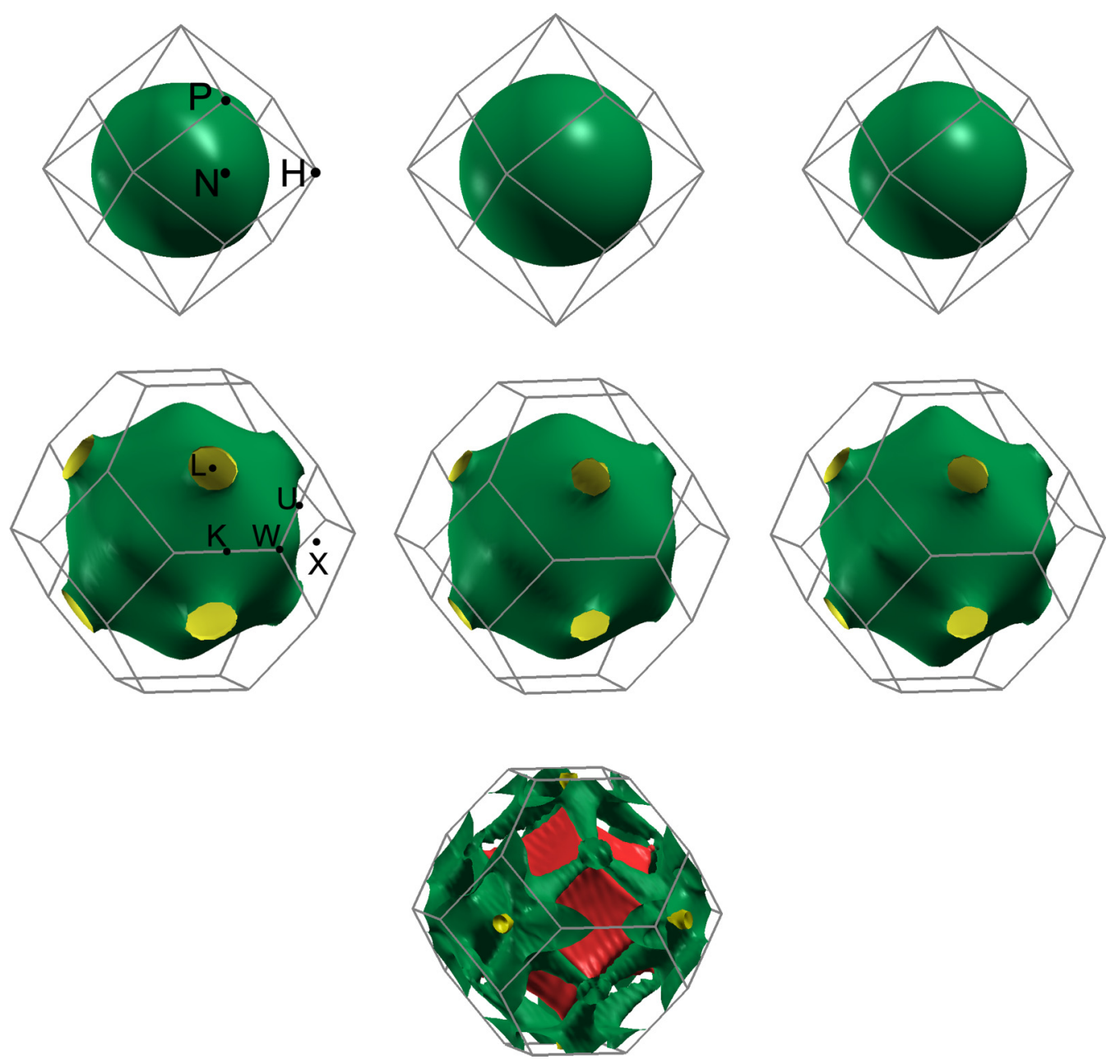

FIG. 3. Calculated Fermi surfaces of $\mathrm{Li}, \mathrm{Na}, \mathrm{K}, \mathrm{Cu}, \mathrm{Ag}, \mathrm{Au}$, and Pt.

at very low temperature (below $50 \mathrm{~K}$ ), and the measurements are highly nonlinear at higher temperature. Both departures from the Bloch-Grüneisen behavior are probably linked to the pre-liquid phase just below the very low melting temperatures: Stronger fluctuations of density and structure create an additional internal resistivity - the study of the electrical resistance in a unified Boltzmann type formalism across the melting point would be a very interesting topic for future research. For the noble metals, excellent agreement is obtained between calculations and experiments [47-49], for both low and high temperatures, even for Pt with a complex Fermi surface. It is interesting to point out that the transport EPC constants reflect the relative magnitudes of $\rho$ in these metals.

Finally, the temperature dependence of the Seebeck coefficients $S$ are computed with both CRTA and VA approaches and compared with experimental data [47-62], as shown in Fig. 5. It is worth noting that $S$ in metals is a more delicate quantity than $\rho$, due to the cancellation effect from, respectively, the electronlike and holelike charge carriers above and below the Fermi energy [Eqs. (A4) and (A14) in Appendix A]. For the studied alkali metals, Li is the only one that exhibits an abnormal positive sign of $S$. The variational prediction yields not only the correct sign but also a quantitative agreement with experiments. On the contrary, CRTA predicts a negative $S$, implying that the constant relaxation time fails even in a simple monovalent metal such as Li. As it was explained in Ref. [24], the positive $S$ in Li can be attributed to the peak in the DOS near the Fermi energy due to band bending near the edge of the BZ. This leads to a higher scattering rate for electrons above the $\epsilon_{F}$ than holes below the $\epsilon_{F}$. For $\mathrm{Na}$ and $\mathrm{K}$, both methods predict the correct negative sign of $S$; however, the quantitative agreement is excellent for VA, while CRTA largely underestimates the magnitude. Even for metals with normal sign of $S$, VA is a much more accurate method (though certainly heavier numerically) compared with CRTA. The melting transition appears as a jump ( $\mathrm{Li})$ or a kink $(\mathrm{Na})$ of the experimental Seebeck coefficient, as opposed to the divergence in $\rho$.

$\mathrm{Cu}, \mathrm{Ag}$, and $\mathrm{Au}$ are three noble metals that have positive Seebeck coefficients. Again, CRTA predicts wrong signs here, i.e., negative $S$. For VA, excellent agreement is achieved in $\mathrm{Cu}$ at all temperatures. In $\mathrm{Ag}$, there is slight underestimation when compared with the data of Ref. [57] (LB [58Cu]), and moderate underestimation when compared with the data of Ref. [58] (LB [56Ru]). The small "hump" at very low temperature from the Ref. [60] (LB [65Sc]) experimental data is probably caused by phonon drag, which is not included in the present study. Test calculations considering thermal expansion (larger lattice constant) yield larger $S$, suggesting that the agreement can be improved further. In $\mathrm{Au}$, the VA predicted 

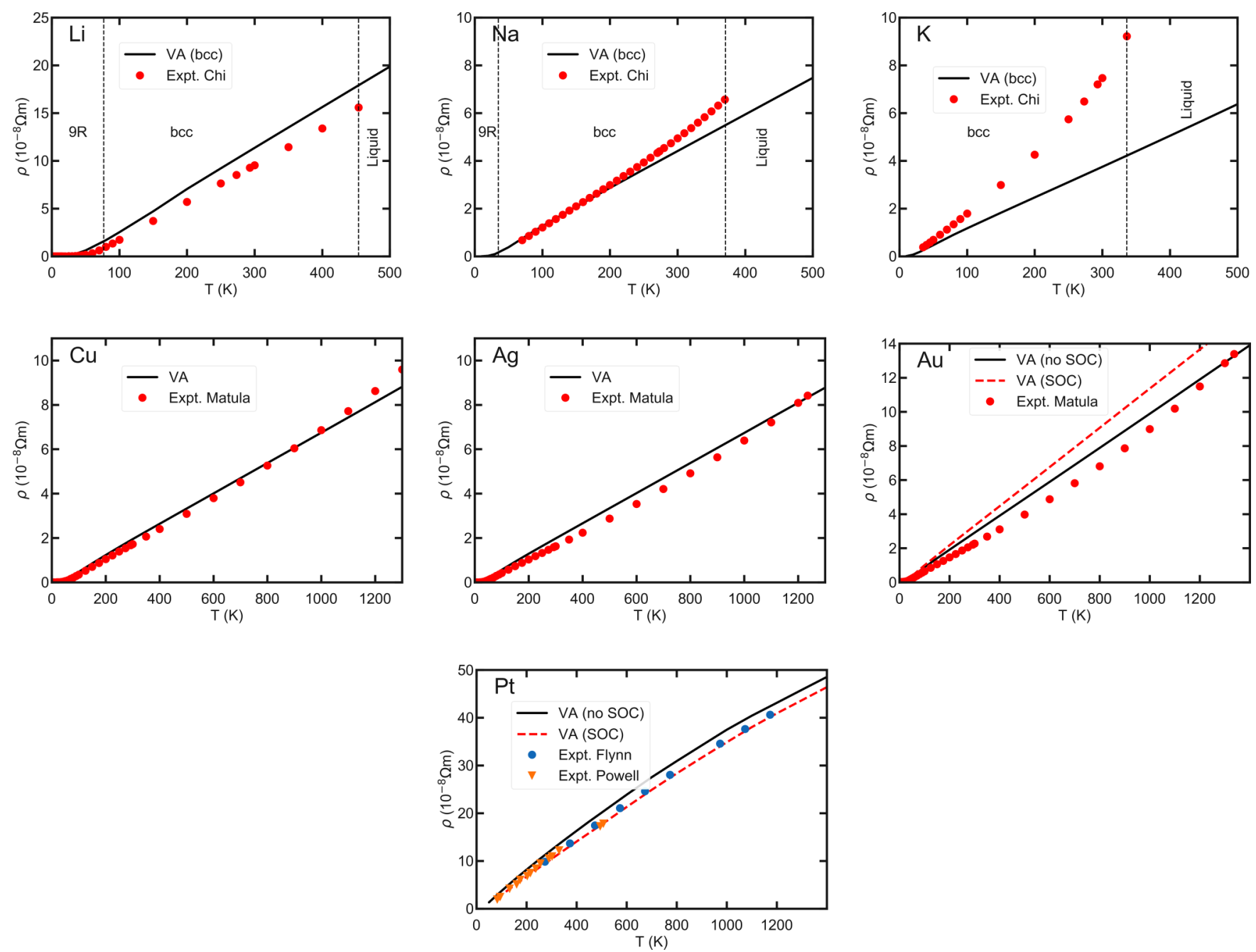

FIG. 4. Electrical resistivity as a function of temperature for $\mathrm{Li}, \mathrm{Na}, \mathrm{K}, \mathrm{Cu}, \mathrm{Ag}, \mathrm{Au}$, and Pt, compared with experimental data [46-49]. For heavy elements $\mathrm{Au}$ and $\mathrm{Pt}$, results with spin orbit coupling (SOC) are also included.

$S$ agrees with experiments for temperature below $\sim 500 \mathrm{~K}$ and overestimates for higher temperatures. Interestingly, a clear saturation of $S$ at high temperature can be noticed in the measured data, which is different from the other studied metals. A possible explanation is that the 'kink' in the electronic structure above $\epsilon_{F}$ in $\mathrm{Au}$ is closer than for $\mathrm{Cu}$ or $\mathrm{Ag}$, and more accurate $G W$ calculations predict that this 'kink' should be even closer [34], which may contribute to the saturation. Including SOC yields a slightly bent curve, with higher values at low temperature and lower for high temperatures, compared with the result without SOC. The shape of the $T$ dependence with SOC has a better agreement with experiment.

For Pt, the measured data are scattered at low temperature, with $S<0$ in Ref. [58] (LB [56Ru]) but $S>0$ below $\sim 200 \mathrm{~K}$ in Refs. [57,62] (LB [73Mo] and [58Cu]). The small positive "hump" again arises due to the phonon drag effect. Ignoring the phonon drag peak, our VA predicted $S$ is always negative and agrees reasonably well with the experimental data. Likely due to the complex Fermi surface, $S$ in Pt has a normal sign, while CRTA wrongly predicts a positive $S$. The several sheets allow many interband $(s-d)$ scatterings involving phonons, which will have very different relaxation times, again underscoring effects beyond the pure electronic dispersion. The
SOC calculations improve the resistivity with respect to experiment but overestimate the Seebeck coefficient-clearly we are pushing the boundaries in accuracy of semilocal DFT band structures with perturbative SOC (in the pseudopotential) and neglecting changes of the band energies with temperature.

\section{CONCLUSIONS}

In summary, we have calculated the electrical resistivity and Seebeck coefficients entirely from a first-principles variational formalism, for selected alkali ( $\mathrm{Li}, \mathrm{Na}$, and $\mathrm{K}$ ) and noble metals $(\mathrm{Cu}, \mathrm{Ag}, \mathrm{Au}$, and $\mathrm{Pt})$, combining electron phonon scatterings, electron band structures, density of states, Fermi surfaces, and phonon dispersion curves. Among these, Li, $\mathrm{Cu}, \mathrm{Ag}$, and $\mathrm{Au}$ have abnormal positive Seebeck coefficients. The theoretical predictions are compared with experimental data, and quantitative agreement is achieved with only a few exceptions, viz., the underestimation of resistivity for $\mathrm{K}$ and $\mathrm{Na}$, understood as a signature of the approaching melting transition. The constant relaxation time approximation is also used to compute the Seebeck coefficients, but it fails to predict the correct sign for $\mathrm{Li}, \mathrm{Cu}, \mathrm{Ag}$, and Au with positive $S$ and for Pt with negative $S$. CRTA also significantly underestimates the 

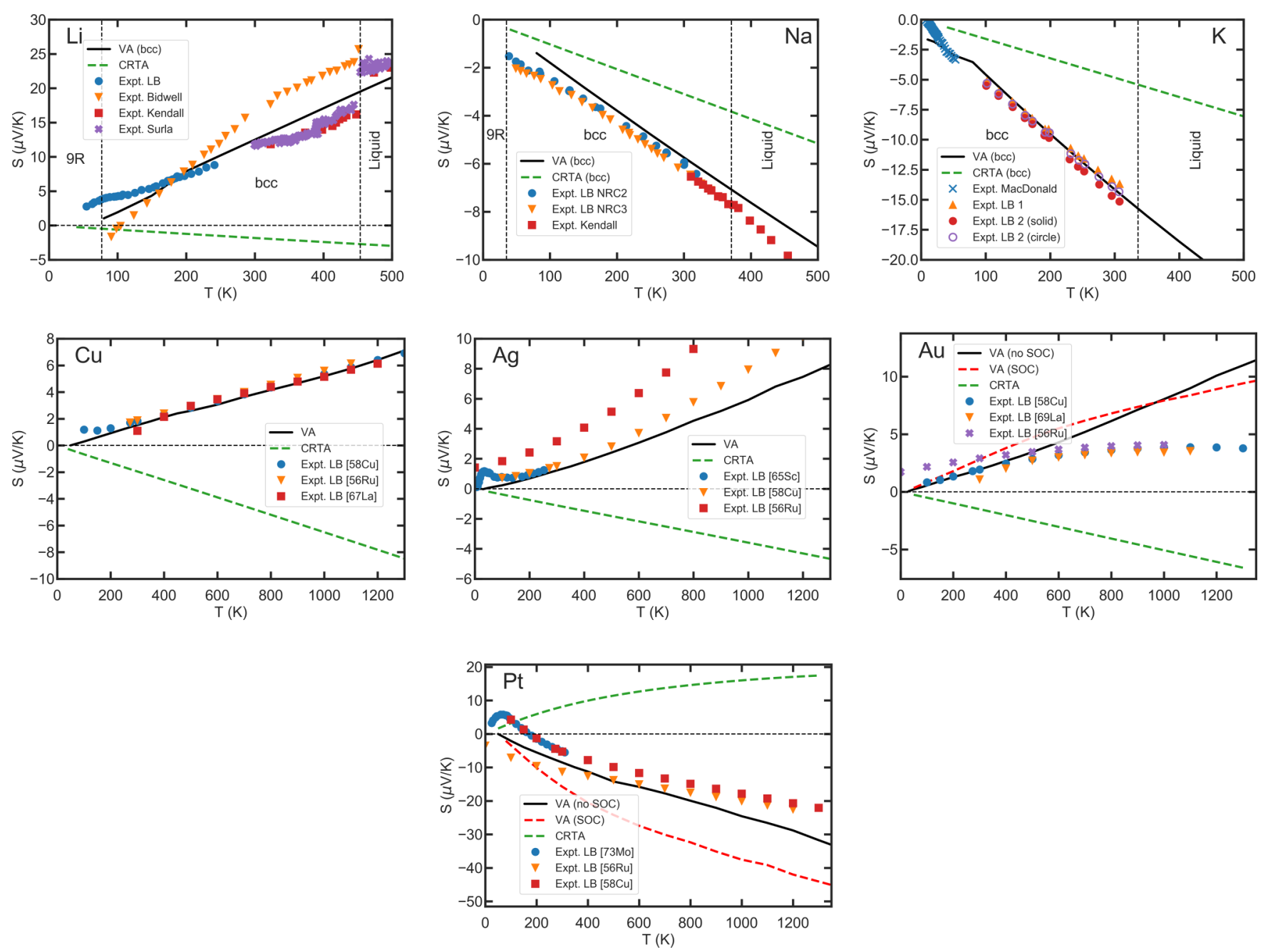

FIG. 5. Seebeck coefficient as a function of temperature for $\mathrm{Li}, \mathrm{Na}, \mathrm{K}, \mathrm{Cu}, \mathrm{Ag}, \mathrm{Au}$, and Pt, compared with experimental data [50-62]. For heavy elements $\mathrm{Au}$ and $\mathrm{Pt}$, results with spin orbit coupling (SOC) are also included.

magnitude of $S$ for $\mathrm{Na}$ and $\mathrm{K}$. We have demonstrated that the variational approach has remarkable accuracy in predicting the transport properties of metals. This includes those with an abnormal sign for the Seebeck effect and complex Fermi surfaces involving $d$ electrons and even for metals with very low $S$ which are numerically challenging. The addition of spin orbit coupling does not seem to improve simultaneously $\rho$ and $S$ for Au and Pt. The SOC effect is probably of the same order of magnitude as other corrections such as anharmonicity and thermal expansion (related to the exceptional ductility of $\mathrm{Au}$ ) and intrinsic exchange correlation errors in the DFT. The case of Au in particular, with high temperature saturation of $S$, warrants further study with even more accurate levels of theory.

\section{ACKNOWLEDGMENTS}

The authors are very grateful for illuminating discussions with P. B. Allen, J. Carrete, and M. Giantomassi. B.X. acknowledges financial support from National Natural Science Foundation of China under Grant No. 12074277, the startup fund from Soochow University, and the support from Priority Academic Program Development (PAPD) of Jiangsu Higher Education Institutions. M.D.G. acknowledges Klanik SA. M.J.V. acknowledges funding by the Belgian FNRS (PDR
G.A. T.1077.15, T.0103.19, and an "out" sabbatical grant to ICN2 Barcelona), and the Fédération Wallonie-Bruxelles (ARC AIMED G.A. 15/19-09). Computational resources have been provided by the Consortium des Equipements de Calcul Intensif (CECI), funded by FRS-FNRS G.A. 2.5020.11, the Zenobe Tier-1 supercomputer funded by the Gouvernement Wallon (G.A. 1117545), and by a PRACE-3IP DECI grants 2DSpin and Pylight on Beskow (G.A. 653838 of Horizon 2020). This publication is based upon work from COST (European Cooperation in Science and Technology) Action TUMIEE (CA17126), and in the framework of the MELODICA project funded by the EU FLAG-ERA JTC2017 call.

\section{APPENDIX A: AB INITIO SEEBECK COEFFICIENT}

\section{Computational details}

We explicitly treat 1 valence electrons for $\mathrm{Li}\left(2 s^{1}\right), \mathrm{Na}$ $\left(3 s^{1}\right)$, and $\mathrm{K}\left(4 s^{1}\right) ; 11$ valence electrons for $\mathrm{Cu}\left(3 d^{10} 4 s^{1}\right), \mathrm{Ag}$ $\left(4 d^{10} 5 s^{1}\right)$, and $\mathrm{Au}\left(5 d^{10} 6 s^{1}\right)$; and 10 valence electrons for $\mathrm{Pt}$ $\left(5 d^{9} 6 s^{1}\right)$. The lattice constants were fully relaxed (stresses below $0.01 \mathrm{GPa}$ ) yielding the lattice constants given in the main text. To check the "purely electronic" SOC effect, the same lattice constants are used, relaxed without SOC. The 
pseudopotentials employed are generated by the FHI-98PP [63] (no SOC) and the Hartwigsen-Goedecker-Hutter codes [64] (SOC).

\section{The Boltzmann transport equation}

In bulk materials, the transport properties of electrons under a uniform electric field $\mathbf{E}$ and temperature gradient $\nabla T$ are often described by the semiclassical Boltzmann's transport equation (BTE), i.e.,

$$
-\mathbf{v}_{\mathbf{k}} \cdot \frac{\partial f_{\mathbf{k}}}{\partial T} \nabla T-\mathbf{v}_{\mathbf{k}} \cdot e \frac{\partial f_{\mathbf{k}}}{\partial \epsilon_{\mathbf{k}}} \mathbf{E}=-\left.\frac{\partial f_{\mathbf{k}}}{\partial t}\right|_{\text {scatt }},
$$

where $e$ is the absolute value of the charge of an electron, $\mathbf{v}_{\mathbf{k}}$ is the group velocity at state $\mathbf{k}$ (short for $\mathbf{k} n$, wave number and band index), $\epsilon_{\mathbf{k}}$ is the energy of the electron at state $\mathbf{k}$, and $f_{\mathbf{k}}$ is the probability distribution function at state $\mathbf{k}$. The term on the right hand side of Eq. (A1) is due to the scattering of electrons, e.g., with phonons, electrons, impurities, defects, etc.

\section{The relaxation time approximation}

The BTE can be solved by assuming relaxation time that characterizes the average time between scatterings. The partial derivative of the scattering term in Eq. (A1) can be approximated as

$$
-\left.\frac{\partial f_{\mathbf{k}}}{\partial t}\right|_{\text {scatt }}=\frac{f_{\mathbf{k}}-f_{\mathbf{k}}^{0}}{\tau_{\mathbf{k}}}
$$

where $\tau_{\mathbf{k}}$ is the relaxation time that depends on $\mathbf{k}$. In cases that the relaxation time has a weak $\mathbf{k}$ dependence, the calculation of the transport properties can be much simplified with a constant relaxation time. Thus the $\mathbf{k}$ dependence only resides in the band structure. This approach has been adopted by the BOLTZTRAP [11,12] and BOLTZWANN [13] codes. An energy-dependent conductivity tensor can be defined as

$$
\begin{aligned}
\sigma_{\alpha \beta}(\epsilon) & =N_{s} e^{2} \sum_{\mathbf{k}} \tau_{\mathbf{k}} v_{\alpha}(\mathbf{k}) v_{\beta}(\mathbf{k}) \delta\left(\epsilon-\epsilon_{\mathbf{k}}\right) \\
& \rightarrow N_{s} e^{2} \tau \sum_{\mathbf{k}} v_{\alpha}(\mathbf{k}) v_{\beta}(\mathbf{k}) \delta\left(\epsilon-\epsilon_{\mathbf{k}}\right),
\end{aligned}
$$

where $N_{s}$ is a spin factor of 2 if the bands are spin degenerate and not spinors. In the collinear spin case there are $2 \sigma$ tensors, one for each spin. The Seebeck coefficient can be written as

$$
\begin{aligned}
S_{\alpha \beta}= & \frac{1}{e T} \frac{\int \sigma_{\alpha \beta}(\epsilon)\left(\epsilon-\epsilon_{F}\right)\left(-\frac{\partial f}{\partial \epsilon}\right) d \epsilon}{\int \sigma_{\alpha \beta}(\epsilon)\left(-\frac{\partial f}{\partial \epsilon}\right) d \epsilon} . \quad \text { (A4) } \begin{array}{l}
\text { functions, the scattering operators in Eq. (A6) are calculat } \\
\text { as: }
\end{array} \\
\left(Q_{n n^{\prime}}\right)_{\alpha \beta}= & \frac{2 \pi V_{\text {cell }} N\left(\epsilon_{\mathrm{F}}\right)}{\hbar k_{\mathrm{B}} T} \int d \epsilon d \epsilon^{\prime} d \omega \sum_{s, s^{\prime}= \pm 1} f(\epsilon)\left[1-f\left(\epsilon^{\prime}\right)\right] \times\left\{[n(\omega)+1] \delta\left(\epsilon-\epsilon^{\prime}-\hbar \omega\right)+n(\omega) \delta\left(\epsilon-\epsilon^{\prime}+\hbar \omega\right)\right\} \\
& \times \alpha_{\text {Allen }}^{2} F\left(s, s^{\prime}, \alpha, \beta, \epsilon, \epsilon^{\prime}, \omega\right) \times J\left(s, s^{\prime}, n, n^{\prime}, \epsilon, \epsilon^{\prime}\right) .
\end{aligned}
$$

Here $V_{\text {cell }}$ is the volume of the unit cell, $\hbar$ is the reduced Planck constant, $n$ is the Bose Einstein distribution, and $\omega$ is the phonon frequency. $\alpha_{\text {Allen }}^{2} F$ and $J$ are, respectively, the transport spectral function and the joint energy polynomial defined as

$$
\begin{aligned}
\alpha_{\text {Allen }}^{2} F\left(s, s^{\prime}, \alpha, \beta, \epsilon, \epsilon^{\prime}, \omega\right)= & \frac{1}{2 N\left(\epsilon_{F}\right)} \sum_{\mathbf{k} \mathbf{k}^{\prime}}\left|g_{\mathbf{k} \mathbf{k}^{\prime}}\right|^{\mathbf{q} v} \delta\left(\epsilon_{\mathbf{k}}-\epsilon\right) \delta\left(\epsilon_{\mathbf{k}^{\prime}}-\epsilon^{\prime}\right) \delta\left(\omega_{\mathbf{q}}-\omega\right) \\
& \times\left[F_{\alpha}(\mathbf{k})-s F_{\alpha}\left(\mathbf{k}^{\prime}\right)\right]\left[F_{\beta}(\mathbf{k})-s^{\prime} F_{\beta}\left(\mathbf{k}^{\prime}\right)\right]
\end{aligned}
$$


with $g_{\mathbf{k k}^{\prime}}^{\mathbf{q} v}=\vec{\eta}_{\mathbf{q} v} / \sqrt{2 M \omega_{\mathbf{q} v}}\left\langle\psi_{k}\left|\delta V^{\mathbf{q}^{v}}\right| \psi_{k^{\prime}}\right\rangle$ being the electronphonon coupling (EPC) matrix between electrons at $\mathbf{k}$ at $\mathbf{k}^{\prime}$, through phonon mode $v$ at point $\mathbf{q} . \mathbf{k}^{\prime}-\mathbf{k}=\mathbf{q}+\mathbf{g}$ with $\mathbf{g}$ a reciprocal lattice vector. We omit the sums over electron bands, which are systematically carried out along with the sum over wave vectors $\mathbf{k}$ and $\mathbf{k}^{\prime}$. Both intra and interband transitions are taken into account in all cases. $M$ is the atomic mass, and $\vec{\eta}_{\mathbf{q} v}$ are the phonon eigenvectors. $\delta V^{\mathbf{q} v}$ is the deformation potential. $\psi_{\mathbf{k} j}$ is the wave function for state $\mathbf{k} j$. Here only the scattering of electrons by phonons is considered. The transport spectral function $\alpha_{\text {Allen }}^{2} F$ is analogous to the Eliash- berg spectral function for superconductivity but weighted by contributions from electron velocities.

In analogy to the superconducting EPC constant, a transport EPC constant can be defined as following, which can characterize the strength of EPC relevant to transport.

$$
\lambda_{\mathrm{tr}}=2 \int_{0}^{\infty} d \omega \frac{\alpha_{\mathrm{tr}}^{2} F(\omega)}{\omega}
$$

where the (usual, adiabatic, or on-shell) transport spectral function $\alpha_{\mathrm{tr}}^{2} F(\omega)$ is:

$$
\alpha_{\mathrm{tr}}^{2} F(\omega)=N\left(\epsilon_{F}\right) \frac{\sum_{\mathbf{k} \mathbf{k}^{\prime}}\left|g_{\mathbf{k} j \mathbf{k}^{\prime} j^{\prime}}^{\mathbf{q} v}\right|^{2}\left(v_{\mathbf{k} \alpha}-v_{\mathbf{k}^{\prime} \alpha}\right)^{2} \delta\left(\epsilon_{\mathbf{k}}-\epsilon_{F}\right) \delta\left(\epsilon_{\mathbf{k}^{\prime}}-\epsilon_{F}\right) \delta\left(\omega_{\mathbf{q}}-\omega\right)}{\sum_{\mathbf{k} \mathbf{k}^{\prime}}\left(v_{\mathbf{k} \alpha}-v_{\mathbf{k}^{\prime} \alpha}\right)^{2} \delta\left(\epsilon_{\mathbf{k}}-\epsilon_{F}\right) \delta\left(\epsilon_{\mathbf{k}^{\prime}}-\epsilon_{F}\right)} .
$$

For the sign of the Seebeck coefficient $S$ a crucial quantity is the joint function $J\left(s, s^{\prime}, n, n^{\prime}, \epsilon, \epsilon^{\prime}\right)$ in Eq. (A9):

$$
\frac{1}{4}\left[\frac{\zeta_{n}(\epsilon)}{N(\epsilon) v(\epsilon)}+s \frac{\zeta_{n}\left(\epsilon^{\prime}\right)}{N\left(\epsilon^{\prime}\right) v\left(\epsilon^{\prime}\right)}\right]\left[\frac{\zeta_{n^{\prime}}(\epsilon)}{N(\epsilon) v(\epsilon)}+s^{\prime} \frac{\zeta_{n^{\prime}}\left(\epsilon^{\prime}\right)}{N\left(\epsilon^{\prime}\right) v\left(\epsilon^{\prime}\right)}\right]
$$

With the electric and heat currents [Eq. (A7)], and assuming $Q_{n n^{\prime}}^{-1}$ can be truncated by keeping only the upper left $2 \times 2$ part of the matrix, the electronic transport coefficients can be found as

$$
\begin{aligned}
\rho_{\alpha \beta} & =\frac{1}{2 e^{2}\left(Q_{00}^{-1}\right)_{\alpha \beta}} \approx \frac{1}{2 e^{2}}\left(Q_{00}\right)_{\alpha \beta} \\
S_{\alpha \beta} & =-\frac{\pi k_{B}}{\sqrt{3} e} \frac{\left(Q_{01}^{-1}\right)_{\alpha \beta}}{\left(Q_{00}^{-1}\right)_{\alpha \beta}} \approx \frac{\pi k_{\mathrm{B}}}{\sqrt{3} e} \sum_{\gamma}\left(Q_{01}\right)_{\alpha \gamma}\left(Q_{11}^{-1}\right)_{\gamma \beta} .
\end{aligned}
$$

\section{Umklapp and normal processes}

By wrapping the wave vectors into the first Brillouin zone, we treat all transitions on the same footing and the expressions above are universal. We detail here briefly the distinctions which are made between Umklapp and normal scattering, and some possible pitfalls. It has been traditional since Peierls to separate scattering processes into normal and Umklapp, depending on whether or not the sum of wave vectors goes outside the first Brillouin zone, i.e., whether $\mathbf{g}=0$ in the quasimomentum conservation above, and to consider that only Umklapp processes are resistive both for electron and phonon transport. It has been shown by several authors that this distinction is not universal: As noted by Maznev and Wright [66] the $\mathrm{U} / \mathrm{N}$ separation is ambiguous and depends on the choice of unit cell. Further, this distinction is insufficient: Normal processes can be resistive and Umklapp processes are not always resistive (in the sense that the thermal current can keep the same direction).

In the example of phonon scattering, with an isotropic Debye approximation, $\omega_{\mathbf{q}}=v_{s}|\mathbf{q}|$ and the group velocity is $\mathbf{v}_{g}(\mathbf{q})=\nabla_{\mathbf{q}} \omega_{\mathbf{q}}=v_{s} \hat{\mathbf{q}}$. In this case

$$
\begin{gathered}
\mathbf{q}+\mathbf{q}^{\prime}=\mathbf{q}^{\prime \prime} \rightarrow \\
v_{s}|\mathbf{q}| v_{s} \hat{\mathbf{q}}+v_{s}\left|\mathbf{q}^{\prime}\right| v_{s} \hat{\mathbf{q}}^{\prime}=v_{s}\left|\mathbf{q}^{\prime \prime}\right| v_{s} \hat{\mathbf{q}}^{\prime \prime} \\
\omega_{\mathbf{q}} \mathbf{v}_{g}(\mathbf{q})+\omega_{\mathbf{q}^{\prime}} \mathbf{v}_{g}\left(\mathbf{q}^{\prime}\right)=\omega_{\mathbf{q}^{\prime \prime}} \mathbf{v}_{g}\left(\mathbf{q}^{\prime \prime}\right) .
\end{gathered}
$$

Here it is clear that the heat current is conserved if $\mathbf{g}=0$. In the general case with band dispersion, however, conservation of quasimoment is different from conservation of current flow (as noted in the hypotheses of Ziman [65] chapter VIII which presumes a strict Debye dispersion).

A counterintuitive phonon case is shown in the cartoon of Fig. 6: A normal process combining the same phonon twice conserves both energy and momentum, stays within the first Brillouin zone (1BZ), and yet arrives in a segment of the phonon dispersion with negative group velocity, producing a heat current in the opposite direction. The process is normal and yet resistive. The inverse can happen with a slightly larger q which goes beyond the 1BZ: It will arrive in the 2BZ (Umklapp process) at a point which has positive group velocity and hence does not backscatter the velocity, though usually the final velocity will be lower and the process is still resistive.

More common (due to energy conservation) are normal transitions combining two acoustic modes into a low lying

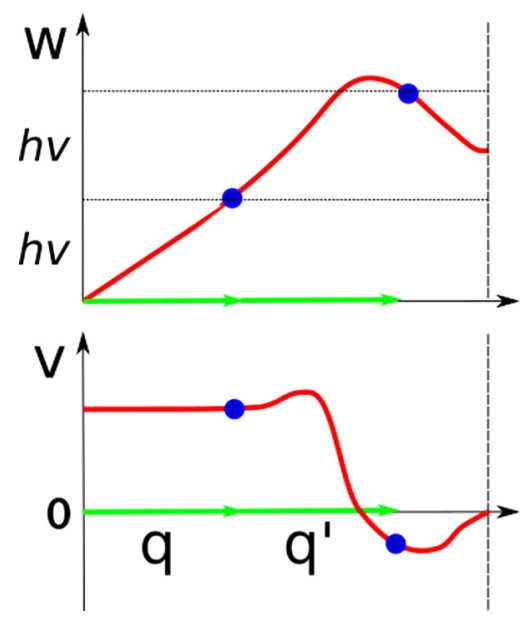

FIG. 6. Example dispersion with a "normal" two phonon process which produces a negative thermal current contribution and is therefore resistive. 


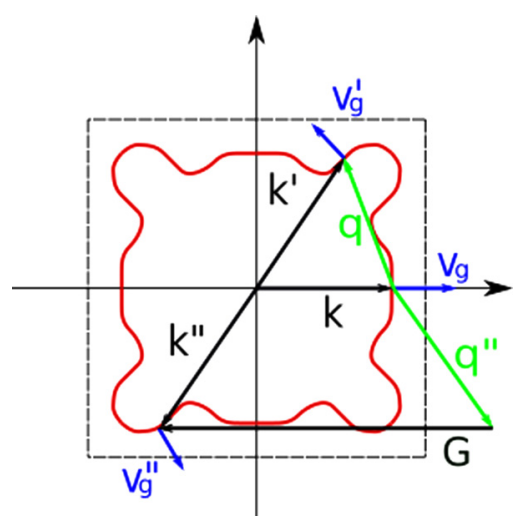

FIG. 7. Example Fermi surface with a "normal" phonon process which produces a negative (resistive) current contribution (along $x$ ) and an Umklapp process which does not backscatter the charge current (remains positive along $x$ ) but only deflects it.

optical one, with much lower group velocity, often negative, at the edge of the 1BZ. Again there is a reduction or even inversion in the thermal current contribution (resistive).
A similar qualitative reasoning is often applied for elastic scattering of electrons in an isotropic parabolic band, by Debye phonons, but suffers from the same limitations. $\mathbf{v}_{g}(\mathbf{k})=$ $\frac{1}{\hbar} \nabla_{\mathbf{k}} \epsilon_{\mathbf{k}}=\frac{\hbar^{2}}{m^{*}} \mathbf{k}$ and all electrons stay at the Fermi level $\epsilon_{F}$. Even in this model, for realistic electron phonon coupling, there is resistivity associated both to $\mathrm{N}$ and $\mathrm{U}$ processes (see, e.g., Ziman [65] chapter IX), as the charge current will always be changed by the scattering. Intuition would still hold that it is changed more by $\mathrm{U}$ processes. However, any nonparabolic dispersion destroys the link between $\mathbf{v}_{g}(\mathbf{k})$ and $\mathbf{k}$, and for "gnarly" Fermi surfaces the velocity can be arbitrarily oriented with respect to $\mathbf{k}: \mathbf{v}_{g}$ and $\mathbf{k}$ can be orthogonal and even antiparallel for a nonspherical Fermi surface (cartoon Fig. 7). Thus a "normal" can generate not just a reduction but a negative contribution to the current, and a resistive Umklapp transition can preserve a positive current contribution.

\section{APPENDIX B: PHONON DISPERSION}

As an essential ingredient of the electron-phonon coupling, DFPT calculated phonon dispersion curves of the alkali and
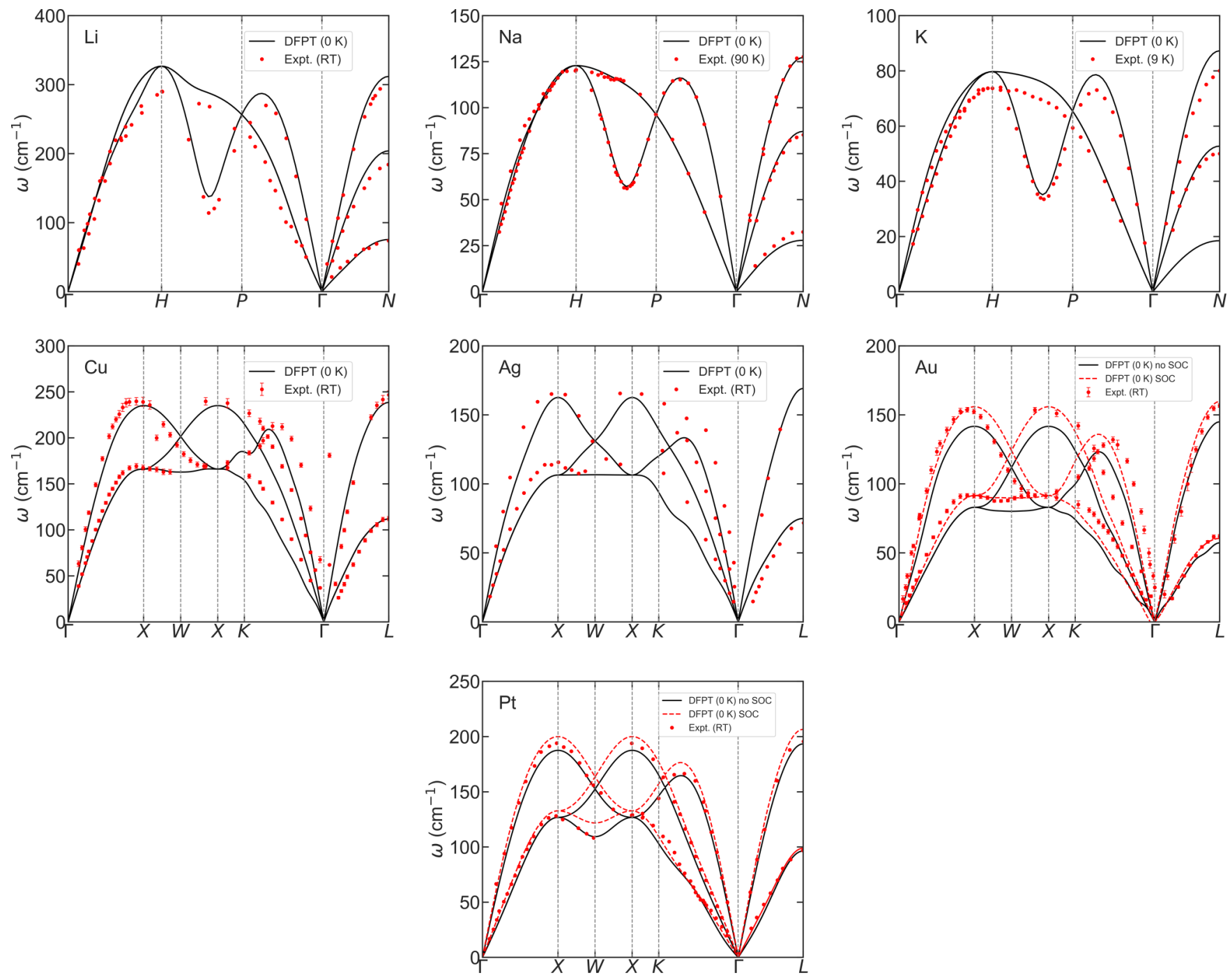

FIG. 8. DFPT calculated phonon dispersion curves, compared with experimental data [67-73], for Li, Na, K, Cu, Ag, Au, and Pt. For heavy elements $\mathrm{Au}$ and $\mathrm{Pt}$, results with spin orbit coupling (SOC) are also included. 

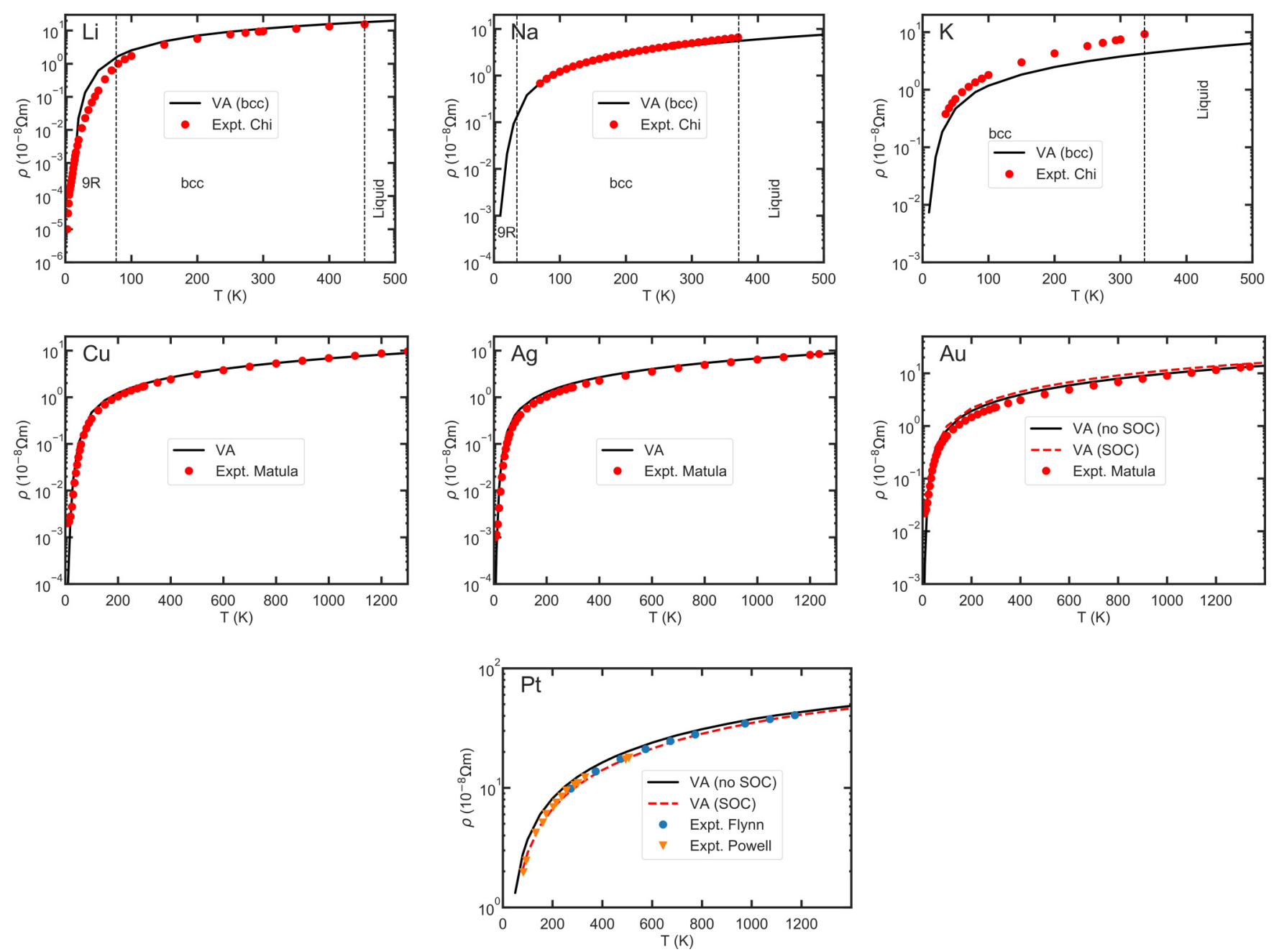

FIG. 9. Logarithmic-scale electrical resistivity as a function of temperature for $\mathrm{Li}, \mathrm{Na}, \mathrm{K}, \mathrm{Cu}, \mathrm{Ag}, \mathrm{Au}$, and Pt, compared with experimental data [46-49]. For heavy elements Au and Pt, results with spin orbit coupling (SOC) are also included.

noble metals are shown in Fig. 8, together with experimental data for comparison. Overall there is very good agreement of our calculations with measured dispersion in all cases (within a few percent for all frequencies). The calculated phonons have a slight overestimation for the alkali metals and slight underestimation for the noble metals, which can be attributed to the DFT lattice constants (Table I), i.e., smaller for alkali metals and larger for noble metals. In general GGA produces larger lattice constants, and additional refinement with semicore electrons may produce even better agreement for the alkalis.

\section{APPENDIX C: LOW-TEMPERATURE ELECTRICAL RESISTIVITY}

To better illustrate the low-temperature electrical resistivity comparison with experiments, we include here logarithmicscale plots for all the studied metals (Fig. 9). The general agreement is rather good above $20 \mathrm{~K}$, considering that $\mathrm{Li}$ and $\mathrm{Na}$ are not bcc below $\sim 77$ and $\sim 35 \mathrm{~K}$, respectively. At lower temperatures, i.e., below $20 \mathrm{~K}$, the numerical integration would require finer $\mathbf{k}$ grids to obtain converged results, and the experimental data is complicated by electron-electron scattering and impurity effects.
[1] K. Behnia, Fundamentals of Thermoelectricity (Oxford University Press, Oxford, UK, 2015).

[2] L. D. Hicks and M. S. Dresselhaus, Phys. Rev. B 47, 12727 (1993).

[3] G. D. Mahan and J. O. Sofo, Proc. Natl. Acad. Sci. USA 93, 7436 (1996).

[4] G. J. Snyder and E. S. Toberer, Nat. Mater. 7, 105 (2008).

[5] G. K. Madsen, J. Am. Chem. Soc. 128, 12140 (2006).
[6] W. Chen, J.-H. Pöhls, G. Hautier, D. Broberg, S. Bajaj, U. Aydemir, Z. M. Gibbs, H. Zhu, M. Asta, G. J. Snyder et al., J. Mater. Chem. C 4, 4414 (2016).

[7] R. Li, X. Li, L. Xi, J. Yang, D. J. Singh, and W. Zhang, ACS Appl. Mater. Interfaces 11, 24859 (2019).

[8] M. V. Tabib, O. M. Løvvik, K. Johannessen, A. Rasheed, E. Sagvolden, and A. M. Rustad, in International Conference on Artificial Neural Networks (Springer, 2018), pp. 392-401. 
[9] Y. Iwasaki, I. Takeuchi, V. Stanev, A. G. Kusne, M. Ishida, A. Kirihara, K. Ihara, R. Sawada, K. Terashima, H. Someya et al., Sci. Rep. 9, 2751 (2019).

[10] T. Wang, C. Zhang, H. Snoussi, and G. Zhang, Adv. Funct. Mater. 30, 1906041 (2020).

[11] G. K. Madsen and D. J. Singh, Comput. Phys. Commun. 175, 67 (2006)

[12] G. K. Madsen, J. Carrete, and M. J. Verstraete, Comput. Phys. Commun. 231, 140 (2018).

[13] G. Pizzi, D. Volja, B. Kozinsky, M. Fornari, and N. Marzari, Comput. Phys. Commun. 185, 422 (2014).

[14] J. Yang, H. Li, T. Wu, W. Zhang, L. Chen, and J. Yang, Adv. Funct. Mater. 18, 2880 (2008).

[15] J.-S. Rhyee, K. H. Lee, S. M. Lee, E. Cho, S. I. Kim, E. Lee, Y. S. Kwon, J. H. Shim, and G. Kotliar, Nature (London) 459, 965 (2009).

[16] S. Poncé, W. Li, S. Reichardt, and F. Giustino, Rep. Prog. Phys. 83, 036501 (2020).

[17] T. Sohier, D. Campi, N. Marzari, and M. Gibertini, Phys. Rev. Materials 2, 114010 (2018)

[18] G. Brunin, H. P. C. Miranda, M. Giantomassi, M. Royo, M. Stengel, M. J. Verstraete, X. Gonze, G.-M. Rignanese, and G. Hautier, Phys. Rev. B 102, 094308 (2020).

[19] A. Jain and A. J. H. McGaughey, Phys. Rev. B 93, 081206 (2016).

[20] J. I. Mustafa, M. Bernardi, J. B. Neaton, and S. G. Louie, Phys. Rev. B 94, 155105 (2016).

[21] Z. Tong, S. Li, X. Ruan, and H. Bao, Phys. Rev. B 100, 144306 (2019).

[22] P. B. Allen, Phys. Rev. B 13, 1416 (1976).

[23] A. Eiguren and I. G. Gurtubay, New J. Phys. 16, 063014 (2014).

[24] B. Xu and M. J. Verstraete, Phys. Rev. Lett. 112, 196603 (2014).

[25] C. M. Hurd, The Hall Effect in Metals and Alloys (Plenum Press, New York, 1972).

[26] C. Kittel and P. McEuen, Introduction to Solid State Physics (Wiley, New York, 1976), Vol. 8.

[27] S. Kou and H. Akai, Solid State Commun. 276, 1 (2018).

[28] H. Gomi, and T. Yoshino, Phys. Rev. B 100, 214302 (2019).

[29] X. Gonze, Phys. Rev. B 55, 10337 (1997).

[30] X. Gonze and C. Lee, Phys. Rev. B 55, 10355 (1997).

[31] X. Gonze, G.-M. Rignanese, M. Verstraete, J.-M. Beuken, Y. Pouillon, R. Caracas, F. Jollet, M. Torrent, G. Zerah, M. Mikami, P. Ghosez, M. Veithen, V. Olevano, L. Reining, R. Godby, G. Onida, D. Hamann, and D. C. Allan, Z. Kristallogr. 220, 558 (2005).

[32] X. Gonze, F. Jollet, F. A. Araujo, D. Adams, B. Amadon, T. Applencourt, C. Audouze, J.-M. Beuken, J. Bieder, A. Bokhanchuk et al., Comput. Phys. Commun. 205, 106 (2016).

[33] X. Gonze, B. Amadon, G. Antonius, F. Arnardi, L. Baguet, J.-M. Beuken, J. Bieder, F. Bottin, J. Bouchet, E. Bousquet et al., Comput. Phys. Commun. 248, 107042 (2020).

[34] T. Rangel, D. Kecik, P. E. Trevisanutto, G.-M. Rignanese, H. Van Swygenhoven, and V. Olevano, Phys. Rev. B 86, 125125 (2012).

[35] L. E. Díaz-Sánchez, A. H. Romero, M. Cardona, R. K. Kremer, and X. Gonze, Phys. Rev. Lett. 99, 165504 (2007).

[36] M. J. Verstraete, M. Torrent, F. Jollet, G. Zérah, and X. Gonze, Phys. Rev. B 78, 045119 (2008).
[37] P. Allen, Phys. Rev. B 17, 3725 (1978).

[38] W. P. Davey, Phys. Rev. 25, 753 (1925).

[39] P. B. Allen, Phys. Rev. B 36, 2920 (1987).

[40] G. Grimvall, The Electron-Phonon Interaction in Metals (NorthHolland, Amsterdam, 1981), Vol. 8.

[41] Y. Wang, Z. Lu, and X. Ruan, J. Appl. Phys. 119, 225109 (2016).

[42] R. Berliner, O. Fajen, H. G. Smith, and R. L. Hitterman, Phys. Rev. B 40, 12086 (1989).

[43] Note that the relaxed lattice constants without SOC are adopted for Au and Pt. The SOC effect on band structures and transport properties are purely electronic, while the volume effect is neglected. Including SOC for $\mathrm{Au}$ or $\mathrm{Pt}$ is found to increase the lattice constant by $2 \%$, compared with the relaxed value without SOC.

[44] G.-Y. Guo, S. Murakami, T.-W. Chen, and N. Nagaosa, Phys Rev. Lett. 100, 096401 (2008).

[45] S. Y. Savrasov and D. Y. Savrasov, Phys. Rev. B 54, 16487 (1996).

[46] T. Chi, J. Phys. Chem. Ref. Data 8, 339 (1979).

[47] R. A. Matula, J. Phys. Chem. Ref. Data 8, 1147 (1979).

[48] D. R. Flynn and M. E. O’Hagan, J. Res. Natl. Bur. Stand., Sect. C 71C, 255 (1967).

[49] R. Powell, R. Tye, and M. J. Woodman, Platinum Metals Rev. 6, 138 (1962).

[50] C. L. Foiles, in Electronic Properties Metals: Electronic Transport Phenomena, Electrical Resistivity, Thermoelectrical Power and Optical Properties, edited by K. H. Hellwege and J. L. Olsen, Landolt-Bornstein, New Series, Group III, Vol. 15b (Springer-Verlag, Berlin, Heidelberg, 1985).

[51] C. C. Bidwell, Phys. Rev. 23, 357 (1924).

[52] P. Kendall, Phys. Chem. Liq. 1, 33 (1968).

[53] V. Surla, M. Tung, W. Xu, D. Andruczyk, M. Neumann, D. N. Ruzic, and D. Mansfield, J. Nucl. Mater. 415, 18 (2011).

[54] D. K. C. Macdonald, W. Pearson, and I. Templeton, Proc. R. Soc. London, Ser. A 248, 107 (1958).

[55] J. Cook, M. V. d. Meer, and M. Laubitz, Can. J. Phys. 50, 1386 (1972).

[56] J. Cook and M. Laubitz, in Thermal Conductivity 14, edited by P. G. Klemens and T.K. Chu (Springer Science+Business Media, New York, 1976), pp. 105-111.

[57] N. Cusack and P. Kendall, Proc. Phys. Soc. 72, 898 (1958).

[58] A. A. Rudnitskii, Thermoelectric properties of the noble metals and their alloys, Tech. Rep. AEC-tr-3724, The Publishing House of the Academy of Sciences, USSR, Moscow, 1956.

[59] M. Laubitz, Can. J. Phys. 45, 3677 (1967).

[60] P. Schroeder, R. Wolf, and J. Woollam, Phys. Rev. 138, A105 (1965).

[61] M. Laubitz, Can. J. Phys. 47, 2633 (1969).

[62] J. Moore and R. Graves, J. Appl. Phys. 44, 1174 (1973).

[63] M. Fuchs and M. Scheffler, Comput. Phys. Commun. 119, 67 (1999).

[64] M. Krack, Theor. Chem. Acc. 114, 145 (2005).

[65] J. Ziman, Electrons and Phonons (Oxford University Press, London, 1961).

[66] A. Maznev and O. Wright, Am. J. Phys 82, 1062 (2014).

[67] M. Beg and M. Nielsen, Phys. Rev. B 14, 4266 (1976). 
[68] A. Woods, B. Brockhouse, R. March, A. Stewart, and R. Bowers, Phys. Rev. 128, 1112 (1962).

[69] R. Cowley, A. Woods, and G. Dolling, Phys. Rev. 150, 487 (1966).

[70] E. Svensson, B. Brockhouse, and J. Rowe, Phys. Rev. 155, 619 (1967).
[71] W. Kamitakahara and B. Brockhouse, Phys. Lett. A 29, 639 (1969).

[72] J. Lynn, H. Smith, and R. Nicklow, Phys. Rev. B 8, 3493 (1973).

[73] D. Dutton, B. Brockhouse, and A. Miiller, Can. J. Phys. 50, 2915 (1972). 